\title{
Feature Extraction of Face Image Based on Machine Learning Method under New Media
}

\author{
Xuefei Han \\ Institute of Fashion Design(Image Design), Dalian Art College, Dalian, 116021, China \\ xuefei_han@yeah.net
}

Keywords: TV drama makeup, Aesthetic characteristics, Using characteristic, Dynamic discrimination, New media, Histogram.

\begin{abstract}
In order to study the relationship between TV drama television makeup design and aesthetic subject, explores the interactive of TV drama makeup and popular culture, in the development process of diversified makeup style, this paper uses multimedia software technology to analyze the aesthetic characteristics of TV drama makeup. Along the sight line of dynamic judgment, this paper does sampling and feature attribution on makeup character characteristics, and calculates the contribution value of sampling point on cosmetic result image. The cumulative value is corresponding to the visibility of aesthetic characteristics. Through the entire pixel traversal TV character makeup image characteristics, this paper statistics strong expression histogram of aesthetic characteristics to judge television makeup effect.
\end{abstract}

\section{Introduction}

With the development of the Chinese TV, TV drama has become one of the most influential entertainments due to its fast propagation and wide audience. At the same time, television makeup is paid more and more attention. TV drama makeup as an important part of television beauty picture, it is also one of the important elements of TV dramas aesthetic value [1, 2]. By television makeup as the breakthrough point, this paper use makeup tree makes a judgment on the aesthetic features, and the main process is as follows:

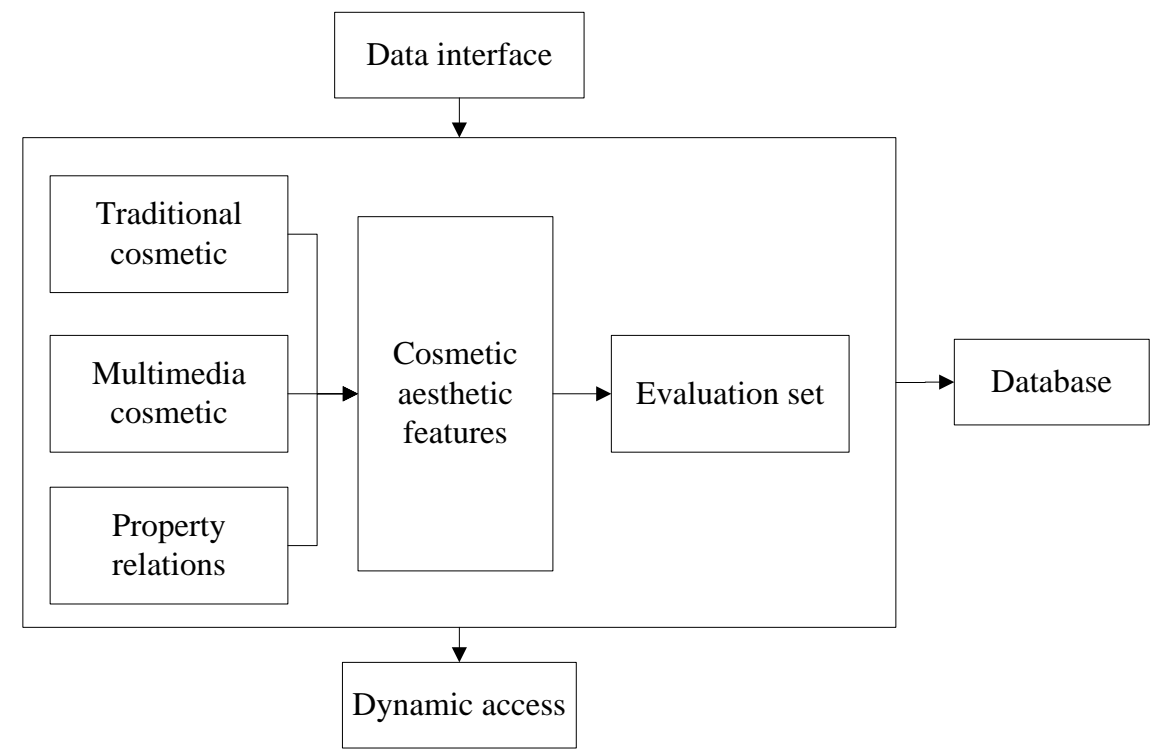

Fig.1: The computer processing framework of makeup

As shown in Figure 1, firstly it uses the traditional makeup and multimedia make-up to design TV character, and multimedia makeup method includes preprocessing makeup before shooting and image processing after the shooting [3]. Through the research on the make-up aesthetic characteristics, it uses database to summary the data information collection, finally the TV drama makeup aesthetic characteristics database is obtained. 


\section{Model and Algorithm Design of Makeup Aesthetic Rate Tree Structure}

Along the sight direction, it can dynamically judge the attribution characteristics of sampling point, and then calculate the contribution value of sampling point on cosmetic result image. The cumulative value is corresponding to the visibility of aesthetic characteristics [4, 5]. Through the entire pixel traversal TV character makeup image characteristics, this paper statistics strong expression histogram of aesthetic characteristics to judge television makeup effect.

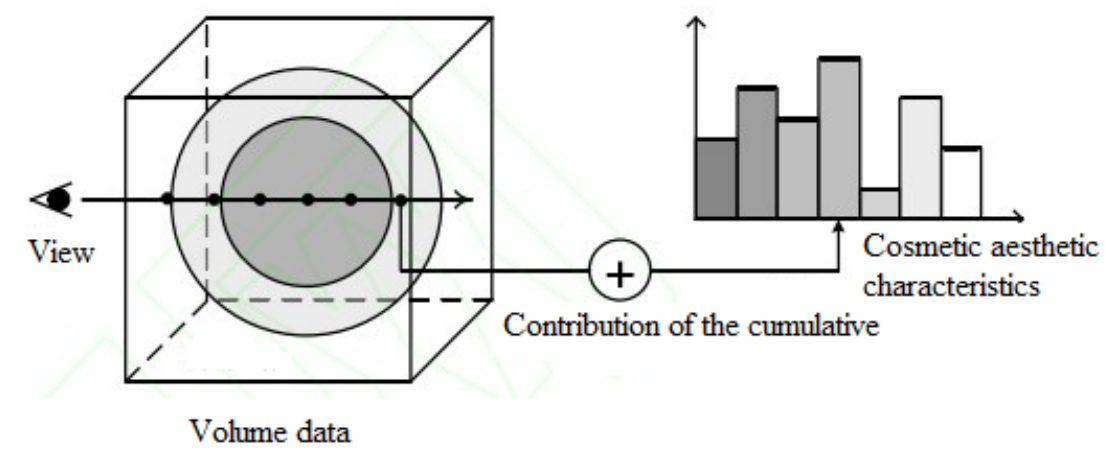

Fig.2: Statistical diagram of cosmetic aesthetic feature visibility histogram

Through the visual histogram of cosmetic aesthetic characteristics, this paper establishes tree modeling and optimizes aesthetic rate feature model [6,7]. The formula of tree structure is as follows:

$$
H(e, f)=\left\{\begin{array}{c}
H\left(f_{e}\right)(e=f) \\
\left.\left.H\left(<H\left(e, \frac{e+f-1}{2}\right), H\right), H\left(\frac{e+f+1}{2}\right), H\right)>\right)(e<f) .
\end{array}\right.
$$

When $e=f$, it uses functions to access data; when $e<f$, the resource is dynamic allocation. Assuming that $S$ is a $i$ dimensional vector, $S=\left[S_{1}, S_{2}, \cdots, S_{i}\right]^{T}$ represents the tree structure network environment, which is equivalent to the branches of tree structure. Assuming that each branch has no crossing as shown in formula (2).

$$
Q(S)=\prod_{i=1}^{n} Q\left(S_{i}\right) .
$$

Tree dynamic structure data can get leaf structure data through linear transformation $Z$, $Z=\left[Z_{1}, Z_{2}, \cdots, Z_{i}\right]^{T}$. It can use the formula (3) to represent its independent component.

$$
Z_{j}=\sum_{i=1}^{n} z_{j i} q_{i}, j=1,2, \ldots, m .
$$

Among them, $z$ is leaf node, $q$ beautify rate coefficient. So the buckling expressions of beautification effect can be written as:

$$
\left([C]+\alpha_{j}[A]\right)\left\{\theta_{j}\right\}=0 .
$$

Among them, $[C]$ is tree stiffness, $\alpha_{j}$ is buckling beautify factor, $[A]$ represents the buckling beautify displacement, $\theta_{j}$ is buckling beautify landscaping. So the expression for any point of the beautification effect is:

$$
R_{i}=\sqrt{\left(\frac{d^{2} H}{d S^{2}}\right)^{2}+\left(\frac{d^{2} Q}{d S^{2}}\right)^{2}+\left(\frac{d^{2} Z}{d S^{2}}\right)^{2}} .
$$

In order to realize the mathematical model of makeup aesthetics, this paper adopts the method of programming algorithm to achieve, and the main program used is as follows [8]:

global T

axes(handles.axes2);

T=getimage; 


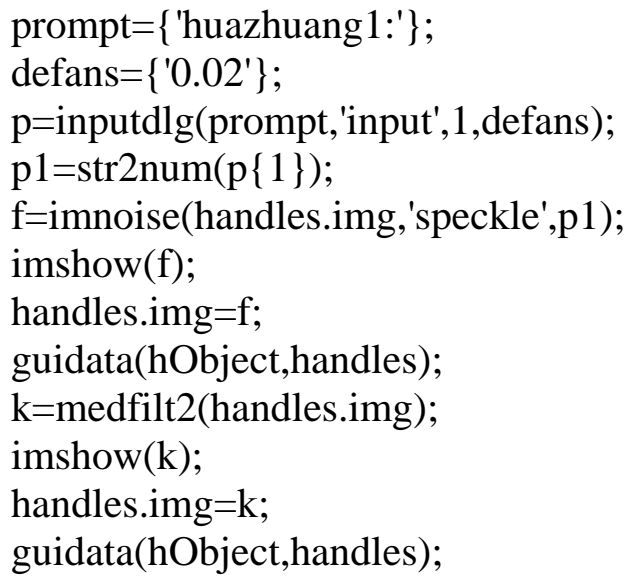

\section{Makeup Aesthetic Characteristics Analysis based on the New Media Software}

In order to verify the effectiveness and reliability of the make-up beautify rate tree model designed in second section, this paper takes the TV drama makeup aesthetic characteristics as an example under the new media software in Beijing, and analyzes the make-up effect of software [9-11]. The basic interface of makeup software is as follows:

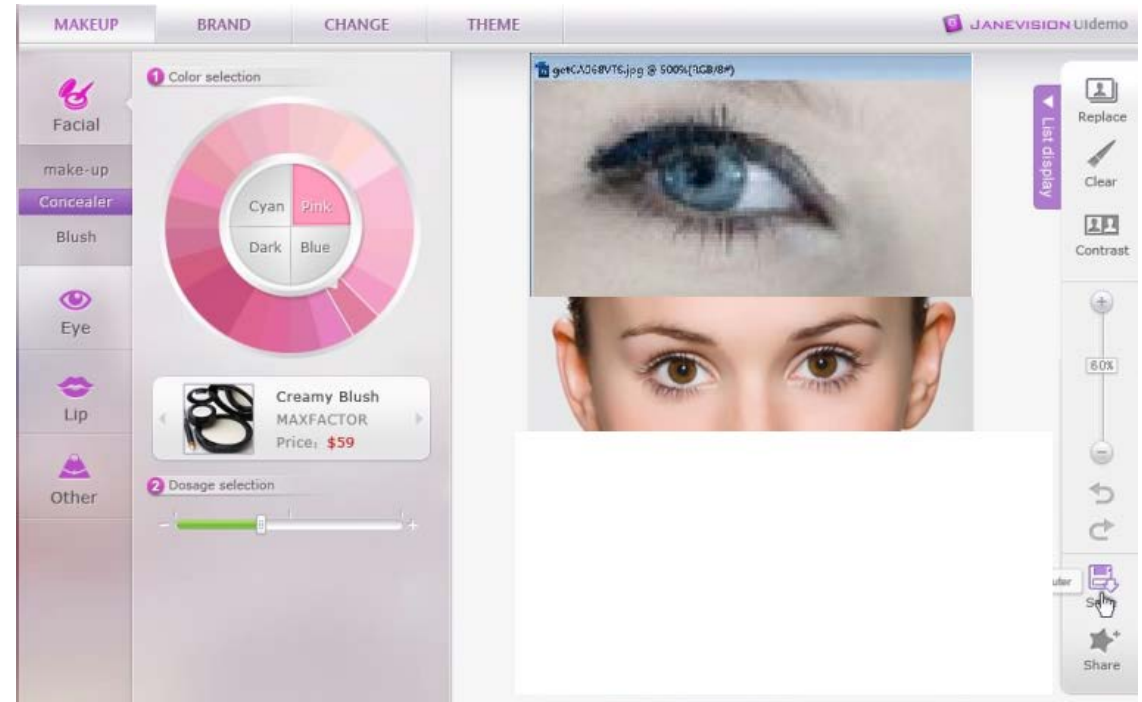

Fig.3: The basic interface of make-up software

Figure 3 shows the basic interface of makeup software [12]. In the new context of media software, make-up pretreatment can use the software to complete, especially characters in TV drama, it can use the software to design image of the characters before shooting, as shown in Figure 4.

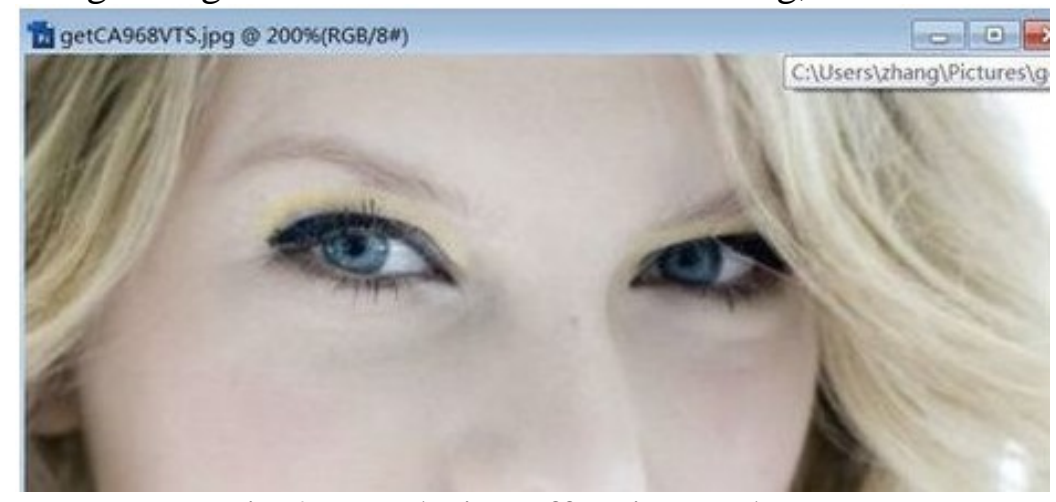

Fig.4: Eye design effect in TV drama 
Figure 4 shows the design effect of character's eyes in TV drama. No matter filming before and after shooting, it can uses software to design the TV film image and optimize the models of the characters. The color optimization is as shown in Figure 5.

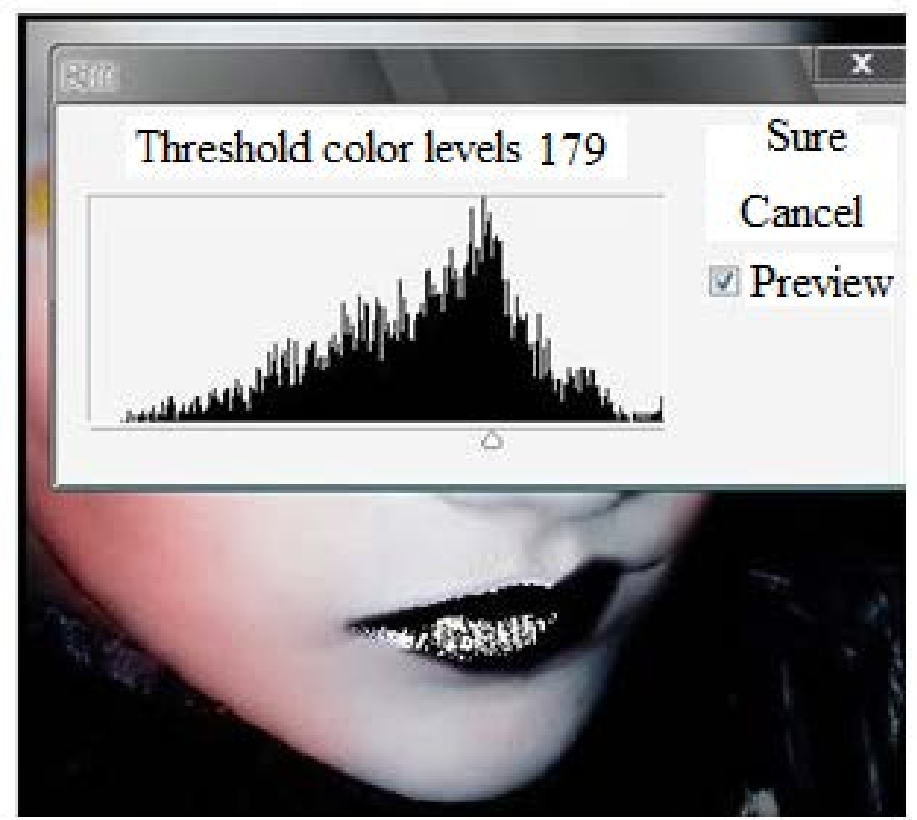

Fig.5: Makeup color level extraction

Figure 5 shows the makeup color level extraction curve in the makeup process [13]. Through the extraction of color gradation signal, it can analyze the aesthetic characteristics and signal characteristics of different frequency band using the color signal frequency and the discretion, as shown in Figure 6.
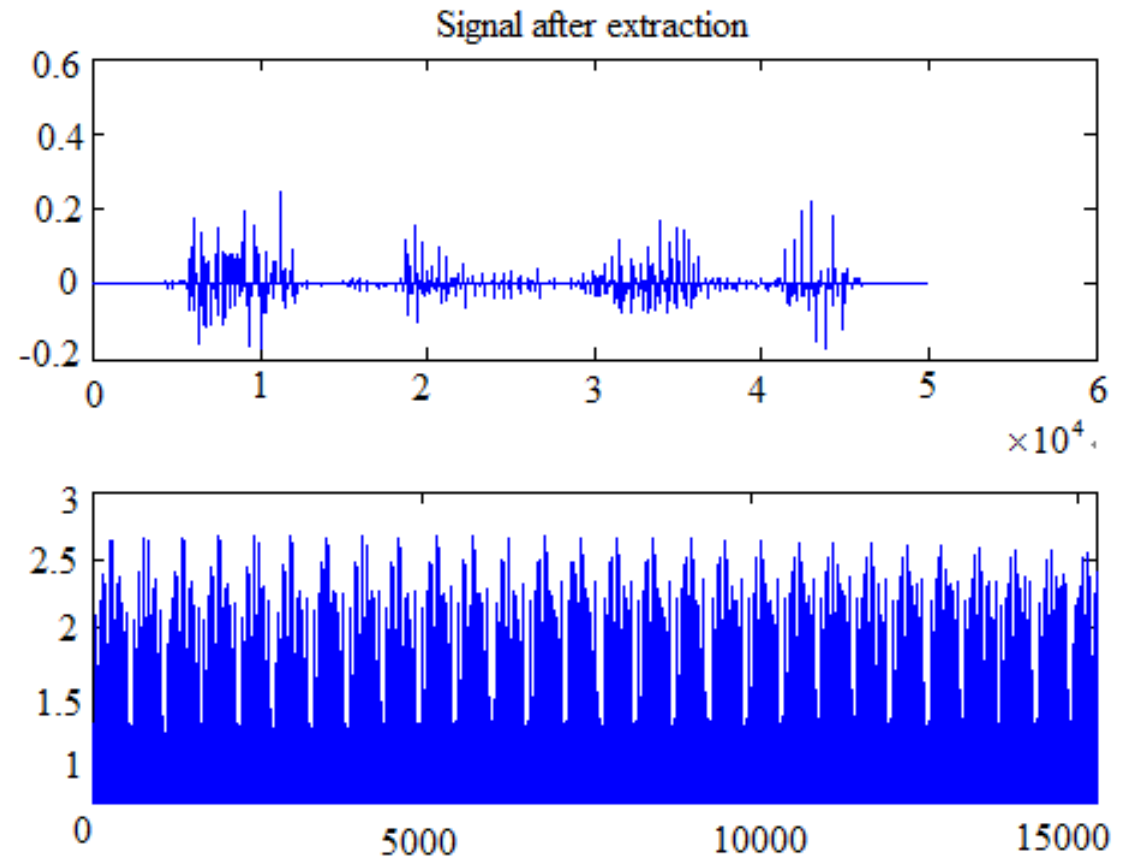

Fig.6: Makeup color signal feature extraction

The above signal is done $1 / 80$ sampling frequency extraction, namely the sampling frequency becomes nearly $500 \mathrm{~Hz}$. As the sampling frequency is relatively small, so the sampling points are sparse, the discrete signal is deviating from the original signal; the spectrum has also undergone aliasing [14]. The beautify rate is statistics and obtains the curve as shown in Figure 7. 


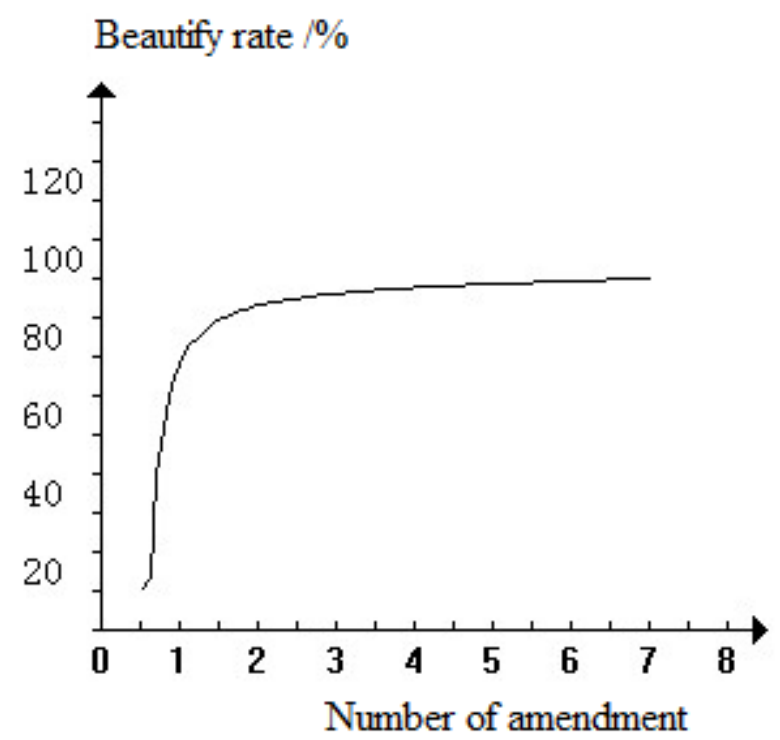

Fig.7: Makeup software beautification effect curve

Figure 7 shows the beautification effect calculation curve of makeup software. From the chart it can be seen, along with the increase of amendment number, beautify rate increased rapidly [15]. When the correction number reached 2, the beautify rate has reached more than $90 \%$, which is in line with the general audience aesthetic characteristics standards. This shows that the tree visualization algorithm is a very makeup efficient algorithm.

\section{Summary}

This paper uses diverse makeup style and multimedia software to analyze aesthetic characteristics of TV drama makeup, and designs the cosmetic landscaping tree visualization algorithm. The algorithm is along the sight direction to dynamically judge makeup character characteristic. Through the contribution of sampling point on makeup structure, analysis of make-up characteristics before and after the TV drama shooting, finally the statistical histogram of TV drama character makeup aesthetic characteristics is obtained. This paper uses the traditional makeup and multimedia make-up to compare the cosmetic properties of TV characters, through multimedia makeup, character makeup color signal extraction curve is obtained. This paper uses signal characteristics to get beautify rate curve changing with revision times, which provides a new multimedia method for television character makeup.

\section{References}

[1] L. Hao. Research status and development of Chinese University fashion show education. Art, 2012, 4(2): 112-115.

[2] B. Lu. The commercial value of fashion show. Popular literature, 2013, 2(4): 24-27.

[3] Y.H. Yu. Application of music in the clothing performing. Big stage, 2012, 3(1): 112-116.

[4] H. Zhang, L.L. Tang Lulu. Optimization of college fashion show professional culture. Big stage, 2014, 2(17): 87-91.

[5] L. Hao. Study on fashion show formation arrangement. Big stage, 2012, 2(4): 91-93.

[6] S.K. Gao. All media concept analysis and theoretical reconstruction. Journal of Zhejiang University of Media and Communications, 2013, 4(9): 41-45.

[7] H.S. Jin, P.L. Tang. TV series network communication strategy. Contemporary film, 2013, 4(20): 76-79.

[8] Y.C. Liu. The characteristics and functions of fashion show background music. North music, 2012, 3(3): 45-48.

[9] H.L. Zhu, L.X. Teng. Analysis of different types of fashion show spread. Flying, 2012, 4(1): 1316. 
[10] Zhu Huanliang, Dong Jianhui. Discussion on the fashion show characteristics. Flying, 2012, 4(5): 61-64.

[11] H.Q. Guo, X.R. Yuan. Transfer functions for volume visualization: state-of-the-art. Journal of Computer-Aided Design \&Computer Graphics, 2012, 24(10):1249-1258.

[12] Y. Wu, H. Qu. Interactive transfer function design based on editing direct volume rendered images. IEEE Transactions on Visualization and Computer Graphics. 2012, 13(5): 1027-1040.

[13] H. Guo, H. Xiao, X. Yuan. Scalable multivariate volume visualizationand analysis based on dimension projection and parallel coordinates. IEEE Transactions on Visualization and Computer Graphic, 2012,18(9):1397-1410.

[14] Lathen G, Lindholm S, Lenz R, et al. Automatic tuning of spatially varying transfer functions for blood vessel visualization. IEEE Transactions on Visualization and Computer Graphics, 2012, 18(12): 2345-2354.

[15] Bruckner S, Möller T. Isosurface similarity maps. Computer Graphics Forum, 2012, 29(3): 773-782. 